\title{
Study on Thixotropic Properties of Waxy Crude Oil Based on Hysteresis Loop Area
}

\author{
Liping Guo, Yu Wang, Shuang Shi, Xiaoyang Yu, Xu Chen \\ College of Petroleum Engineering, Daqing Petroleum Institute, Daqing, China \\ Email: chenxude1990@163.com
}

Received 15 July 2015; accepted 25 July 2015; published 28 July 2015

Copyright (C) 2015 by authors and Scientific Research Publishing Inc.

This work is licensed under the Creative Commons Attribution International License (CC BY). http://creativecommons.org/licenses/by/4.0/

(c) (7) Open Access

\begin{abstract}
Thixotropic properties are one of the low temperature rheology behaviors of gelled waxy crudes. In order to study the thixotropic behavior of gelled waxy crudes oil under cyclic loading of linear increasing and decreasing of shear rate, three different types of waxy crude oil were studied experimentally by using MARSIII rheometer. It is found that hysteresis loop area could represent strength of thixotropic properties under that loading. With increasing of the rate of share rate sweep, the area of second hysteresis loop is much smaller than first one and began a slow decline from the third hysteresis loop. Areas of each hysteresis loops were decreased with increasing temperature, but it was different in decline rate-the lower the temperature, the greater the rate of decline. The maximum rate of decrease is the first hysteresis loop with exponential decline, and remaining hysteresis hoops approximately decrease linearly. In addition, based on the experimental results an empirical correlation is developed to describe the relationship of hysteresis loop area and rate of share rate sweep.
\end{abstract}

\section{Keywords}

Waxy Crude Oil, Thixotropic, Hysteresis Loop, Area

\section{Introduction}

Thixotropy of waxy crude is an important but complex issue in crude oil rheology study. Due to difference between the broken-down structures with the developed structure of fluid system, thixotropy means that apparent viscosity decreases continuously over time under shearing stress, and recovers gradually over time after stress relief [1]. Thixotropy is basic data for shutdown and restart calculation and pumpability evaluation of waxy crude oil pipeline, besides that, safety analysis for running waxy crude oil pipeline needs accurate quantitative description in thixotropic behavior [2]. 
Proposed mathematical modes described thixotropic fluid properties; domestic and foreign scholars have numerous studies in thixotropic fluid. Cheng proposed thixotropic fluid characteristic under three kinds of cyclic shear tests which are constant, stepwise increase and continuously increase in shear rate [3]-[5]. Most of the existing mathematical models proposed are under studying shearing stress relaxation of thixotropic process in a constant shear rate [6] [7] in which Huang put forward mathematical model for blood thixotropy at loading conditions that shear rate changes continuously [8]. Although it has a lot of studies in waxy crude thixotropy, there have not been reports describing change properties of thixotropic hysteresis loop in the loading conditions whose shear rate is continuously variable. However, in the practical crude oil pipeline process, the shear rate is not constant but is continuously variable. In this paper, experiments study the waxy crude oil change properties of thixotropic hysteresis loop area, and result in an empirical correlation to describe the relationship of hysteresis loop area and rate of share rate sweep on basis.

\section{Experiment}

\subsection{Experimental Apparatus and Experimental Samples}

Laboratory instruments are mainly MARSIII rheometer produced by German HAAKE companies, and experiments used its Z41Ti coaxial cylinder measurement system. Rheometer equipped with F6/8 programmable with a water bath temperature controlling precision of $0.1^{\circ} \mathrm{C}$.

Physical properties of four kinds of waxy crude oil used in the experiments are shown in Table 1. To ensure better repeatability and comparability of the experimental data, experimental oil samples were pre-treated to eliminate the historical experience of crude oil "memory" effect. For the basic oil sample, specific operation method is put ground glass bottle filled with sample into the water bath, let stand heated to $80^{\circ} \mathrm{C}$ and constant temperature in $2 \mathrm{~h}$, so that the bottle of crude oil achieve uniform state with the aid of molecular thermal motion, then stand at room temperature for $48 \mathrm{~h}$ above.

\subsection{Experimental Program}

Put ground glass bottle filled with well pretreatment oil sample into the water bath for 30 min, which temperature is the heating temperature of condensation point test on Table 1, and then put the oil sample into the rheometer measuring cylinder and constant temperature for 5 min. When you are making samples, ensure consistent temperature of the oil sample and rheometer system to eliminate the impact of the rheological property once the sample temperature sudden rise or drop. Then cold to measure temperature at rate $0.5^{\circ} \mathrm{C} / \mathrm{min}$, and start measuring after constant temperature stand $40 \mathrm{~min}$ for forming the gelling structure sufficiently. Shear rate changed with time is linear load according to the Equation (1), and the rate of shear rate is respectively $2 \mathrm{~s}^{-1} / \mathrm{s}, 1.5625$ $\mathrm{s}^{-1} / \mathrm{s}, 1.0 \mathrm{~s}^{-1} / \mathrm{s}, 0.5 \mathrm{~s}^{-1} / \mathrm{s}, 0.2 \mathrm{~s}^{-1} / \mathrm{s}, 0.05 \mathrm{~s}^{-1} / \mathrm{s}$ and $0.025 \mathrm{~s}^{-1} / \mathrm{s}$, the rise time of shear rates are respectively $12.5 \mathrm{~s}, 16$ s, 25 s, 50 s, 125 s, 500 s and 1000 s; selected experimental temperature in the vicinity of each sample's condensation point.

$$
\dot{\gamma}=\left\{\begin{array}{cc}
R t & 0 \leq t \leq t_{1} \\
R\left(2 t_{1}-t\right) & t_{1} \leq t \leq 2 t_{1}
\end{array}\right.
$$

where in these equation, $R$ is the rate of shear rate (a constant in experiment), $\mathrm{s}^{-1} / \mathrm{s}$; $t_{1}$ is the shear rate of rise time s.

\section{Results and Analysis}

In this paper, we take experiments of three different physical properties of crude oil, and take experiments totally in 9 temperatures, 6 rate of shear rates. Figure 1 is the experimental curve in a shear rate for $1 \mathrm{~s}^{-1} / \mathrm{s}$.

\section{Table 1. The physical sample property sheet.}

\begin{tabular}{ccccc}
\hline Oil sample & Paraffin content $\mathrm{m} \%$ & Drop out point ${ }^{\circ} \mathrm{C}$ & Condensation point (heating temperature) ${ }^{\circ} \mathrm{C}$ & $20^{\circ} \mathrm{C}$ density $\mathrm{kg} / \mathrm{m}^{3}$ \\
\hline $1^{\#}$ oil sample & 24.37 & 42 & $32(45)$ & 863.11 \\
$2^{\#}$ oil sample & 21.51 & 51 & $33(53)$ & 856.09 \\
$3^{\#}$ oil sample & 14.20 & 44 & $29(50)$ & 866.18 \\
\hline
\end{tabular}




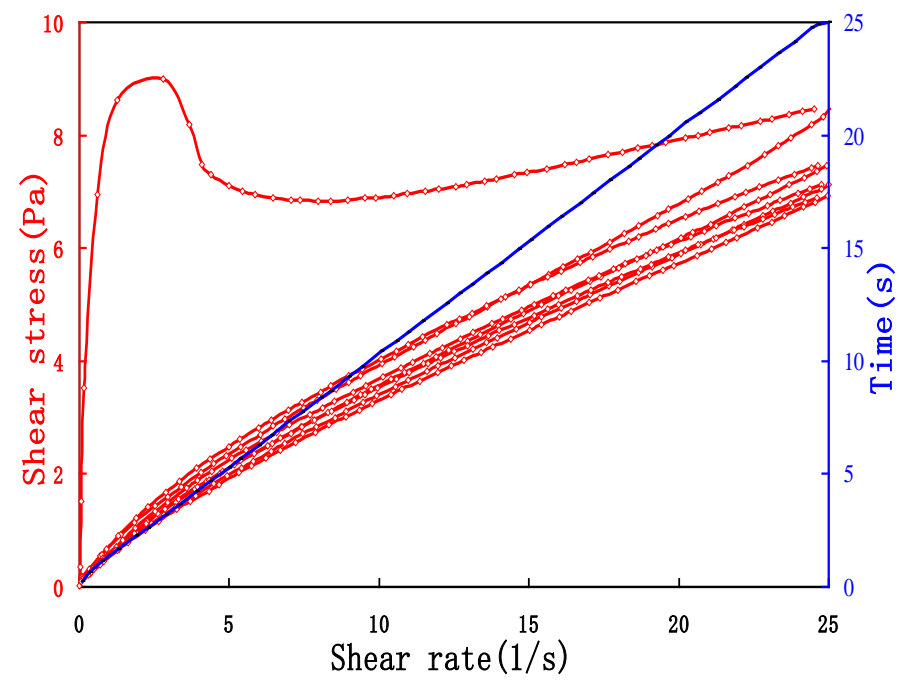

Figure 1. $\mathrm{s}^{-1} 35^{\circ} \mathrm{C}$ experimental curves of Daqing crude oil $\left(\mathrm{R}=1 \mathrm{~s}^{-1} / \mathrm{s}\right)$.

Due to waxy crude oil wax crystal structure has a certain strength in the vicinity of the condensation point, the initial stage of loading sample at a creep process is shown in Figure 1. Performance in hear stress is in rapidly rising stage with the increase of shear rate. After crude yield point, wax crystal structure began pyrolysis and allow it to flow. In the process of shear rate increase upward, with the extension of time and increasing evenly of the shear rate, pyrolysis rate of the internal gelling system wax crystal structure is greater than recovery rate, which makes flow resistance gradually reduced, that is, the shear stress showed a trend of decrease; on the other hand, due to the increase of shear rate, corresponding shear stress exhibits a trend of increase. On the initial stage after yielding, the former plays a leading role, so with the shear rate increases, the shear stress dropped substantially, such as dropped segment curve after the peak in the figure. When the shear rate increases to a certain value, two kinds above have equal role at a particular instant, that is the minimum point of upward curve in the first hysteresis loop in the figure. After that, pyrolysis rate of structure is further reduced, and deformation rate is further increase. The curve transform to the common case that shear stress increases with the increase of shear rate. In the process of shear rate reduce downward, two factors are working together which are the lower the shear rate leads to the decrease of the shear stress and the recovery of wax crystal structure results in shear stress increased, and the former is dominated because of wax crystal slow structure recovery rate. The performance shown in the picture is that shear stress is gradually reduced with the decrease of the shear rate in the downward experimental curve. Under continuing cyclic loading, hysteresis loop area decreases, and move in direction of shear rate axis, but loop area is very small started from the second hysteresis loop, while the thixotropy of system has greatly diminished.

\section{Hysteresis Loop Area to Representation Thixotropic Properties of System}

The size of hysteresis loop can properties the strength of thixotropy in system, this paper calculated area of hysteresis loops for the four kinds of waxy crude oil in different temperatures and different rates of shear rate. Figure 2 is experimental curve comparison chart for different rates of shear rates in $34^{\circ} \mathrm{C}$ for Daqing crude oil. Figure 3 is a curve for area change of hysteresis loop in $32^{\circ} \mathrm{C}$ central plains crude oil. The area of the hysteresis loop increases with the increase of the rate of shear rate. Investigating the reason, in the experimental model of this paper, when the rate of shear rate is greater, the identical shearing action time is shorter; the damage of gelled crude oil system structure is less; the thixotropic structure remain is more; the shear stress is relatively bigger; hysteresis loop has a general tendency to leave shear rate axis direction and the area of the loop is relatively large. Conversely, when the rate of shear rate change small, shear time is relatively long; the extent of damage in wax crystal structure is relatively serious under the same shear rate; shear stress is relatively small; hysteresis loop move back to the shear rate axis's direction; and the area of the loop is relatively small.

Second hysteresis loop area is much smaller than the first hysteresis loop, then the area of loops decline slowly. It illustrates the proportion of structure is damaged large in the shearing progress of first hysteresis loop. 


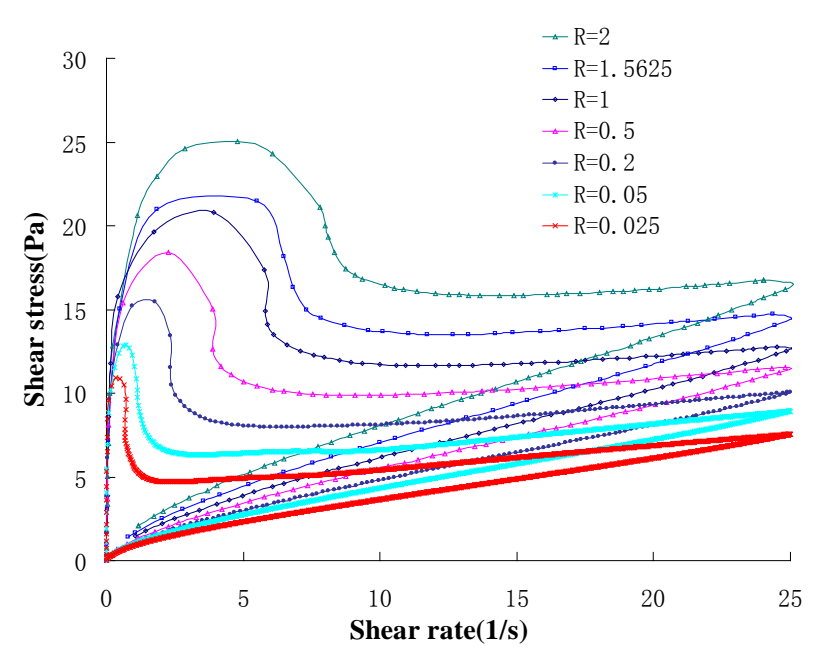

Figure 2. $34^{\circ} \mathrm{C}$ experimental curves of Daqing crude oil under the condition of different rates of shear rate.

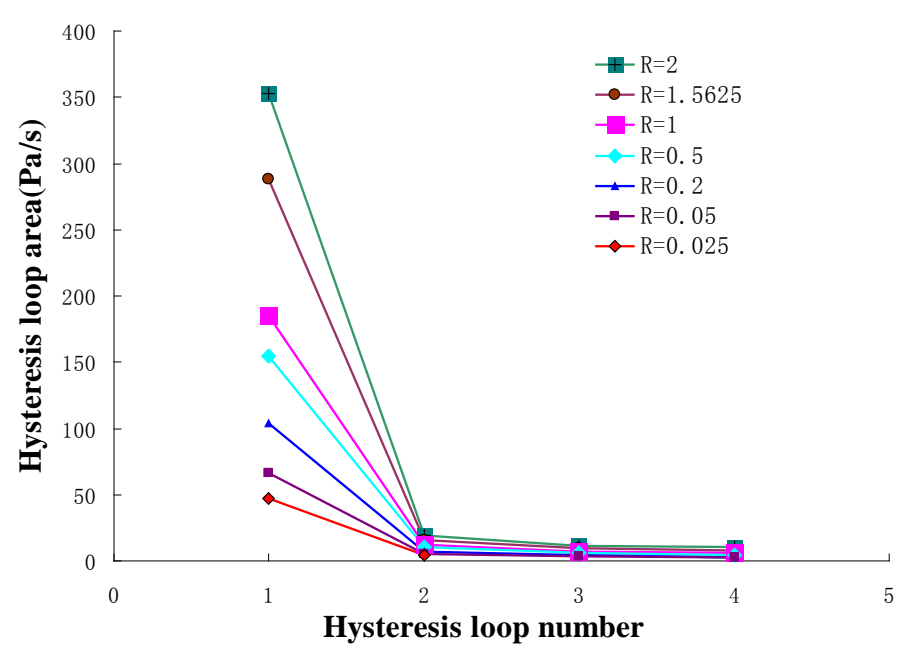

Figure $3.32^{\circ} \mathrm{C}$ experimental curve for area change of hysteresis loop for central plains crude oil.

In order to take quantitative description, statistical ratio of the second with the first hysteresis loop area for three experimental samples in various rate of shear rate are shown in Table 2. Thus we figure out the area of second hysteresis loop is only $10 \%$ of the first loop, which means shear rate and temperature changes have little effect on it. In other words, the structure of the gel volume damaged mainly occurs in the first hysteresis loop.

The ratio of third hysteresis loop relative to the second hysteresis loop area is about $60 \%$ which is shown in Table 3 and the ratio of fourth hysteresis loop relative to the third hysteresis loop area is about $80 \%$ which is shown in Table 4. Which indicates falling speed of hysteresis loop area are reduce, thixotropic structures in oil gelling system are in decline, and also declare crude wax crystal structure of waxy crude oil is recovery slow or incomplete which is consistent with paper findings [9].

Therefore, based on different changes of hysteresis loop area for three kinds of waxy crude physicals at each test temperature, we can summarize Equation (2) from experimental results to indicate the relationship between hysteresis loop area with the rate of shear rate.

$$
A_{n}=a n^{b} \dot{\gamma}^{c}
$$

where in the equation, $A_{n}$ is the $n$-th hysteresis loop area; $a, b, c$ is parameters determined by fitting the experimental data; $\mathrm{n}$ is the hysteresis loop number $(n=1,2,3,4)$. 
Table 2. Ratio of the second with the first hysteresis loop area in various rate of shear rate.

\begin{tabular}{|c|c|c|c|c|c|c|c|c|}
\hline \multirow[t]{2}{*}{ Share rate } & erature & 0.05 & 0.2 & 0.5 & 1 & 1.5625 & 2 & Average \\
\hline & 33 & 0.14 & 0.08 & 0.07 & 0.06 & 0.07 & 0.06 & 0.078 \\
\hline \multirow{3}{*}{$1^{\#}$ oil sample } & 34 & 0.19 & 0.11 & 0.09 & 0.08 & 0.09 & 0.09 & 0.109 \\
\hline & 35 & 0.21 & 0.13 & 0.12 & 0.12 & 0.12 & 0.11 & 0.136 \\
\hline & 36 & 0.25 & 0.15 & 0.14 & 0.14 & & & 0.113 \\
\hline \multicolumn{2}{|c|}{ Average } & 0.196 & 0.118 & 0.104 & 0.101 & 0.093 & 0.087 & \\
\hline \multirow{6}{*}{$2^{\#}$ oil sample } & 31 & 0.07 & 0.06 & 0.06 & 0.04 & 0.04 & 0.12 & 0.065 \\
\hline & 32 & 0.08 & 0.07 & 0.07 & 0.07 & 0.06 & 0.05 & 0.065 \\
\hline & 33 & 0.08 & 0.08 & 0.09 & 0.10 & 0.08 & 0.06 & 0.081 \\
\hline & 34 & 0.10 & 0.11 & 0.12 & 0.12 & 0.11 & 0.10 & 0.110 \\
\hline & 35 & 0.09 & 0.11 & 0.13 & 0.13 & 0.19 & 0.14 & 0.131 \\
\hline & 36 & 0.13 & 0.14 & 0.16 & 0.17 & 0.20 & 0.21 & 0.168 \\
\hline \multirow[t]{3}{*}{ Averag } & & 0.09 & 0.10 & 0.10 & 0.11 & 0.11 & 0.11 & \\
\hline & 28 & 0.06 & 0.05 & 0.05 & 0.05 & & & 0.050 \\
\hline & 29 & 0.06 & 0.06 & 0.06 & 0.05 & 0.05 & 0.05 & 0.054 \\
\hline \multirow[t]{3}{*}{$3^{\#}$ oil sample } & 30 & 0.08 & 0.07 & 0.06 & 0.06 & 0.07 & 0.08 & 0.070 \\
\hline & 31 & 0.11 & 0.09 & 0.09 & 0.09 & 0.11 & 0.13 & 0.104 \\
\hline & 32 & 0.13 & 0.12 & 0.13 & 0.13 & & & 0.085 \\
\hline \multicolumn{2}{|c|}{ Average } & 0.09 & 0.08 & 0.08 & 0.08 & 0.08 & 0.09 & \\
\hline
\end{tabular}

Table 3. Ratio of the third with the second hysteresis loop area in various rate of shear rate.

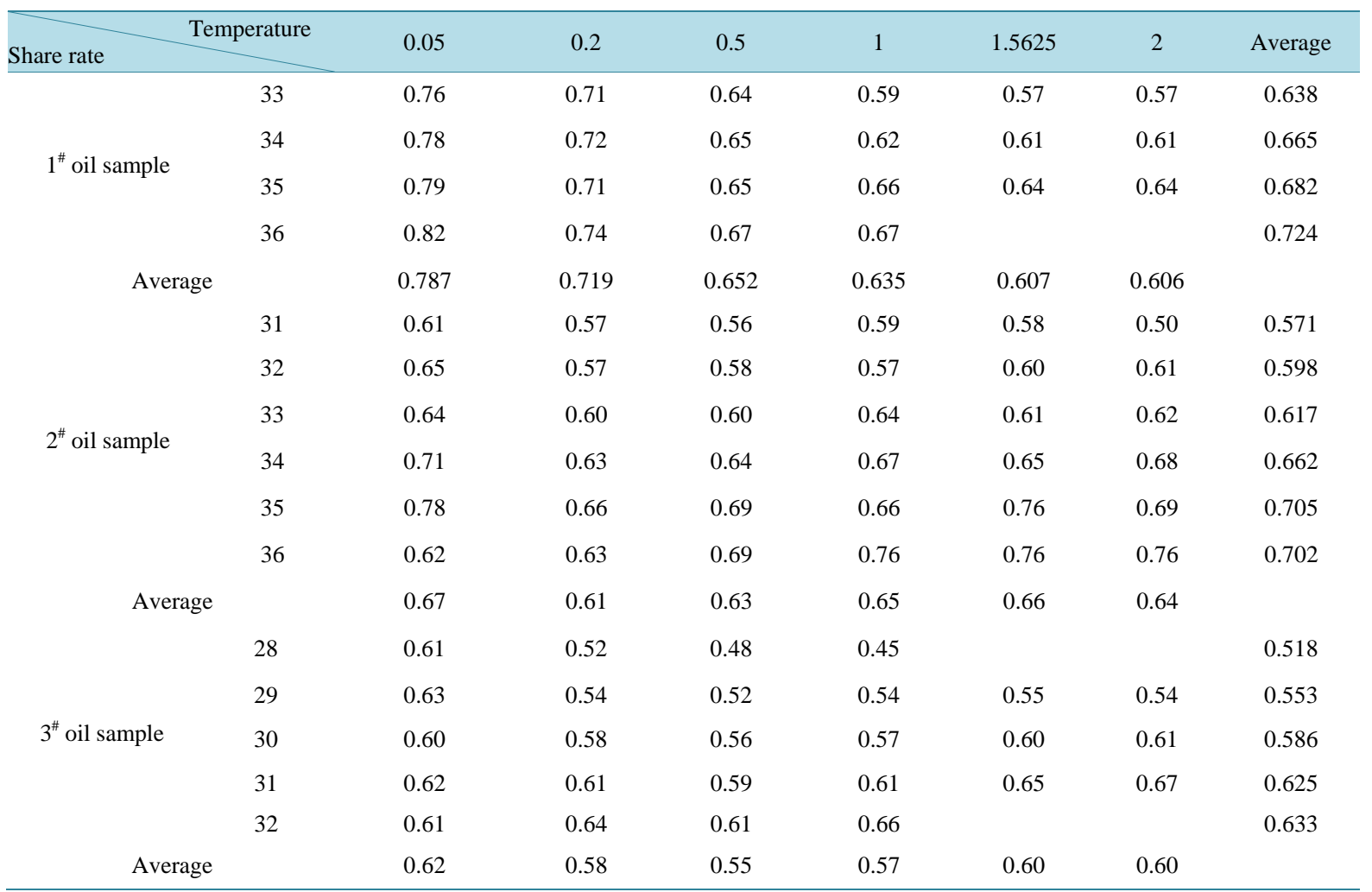


Table 4. Ratio of the fourth with the third hysteresis loop area in various rate of shear rate.

\begin{tabular}{|c|c|c|c|c|c|c|c|c|}
\hline Share rate & Temperature & 0.05 & 0.2 & 0.5 & 1 & 1.5625 & 2 & Average \\
\hline \multirow{4}{*}{$1^{\#}$ oil sample } & 33 & 0.87 & 0.85 & 0.81 & 0.81 & 0.87 & 0.92 & 0.853 \\
\hline & 34 & 0.90 & 0.86 & 0.83 & 0.78 & 0.85 & 0.85 & 0.843 \\
\hline & 35 & 0.91 & 0.86 & 0.82 & 0.78 & 0.81 & 0.88 & 0.843 \\
\hline & 36 & 0.92 & 0.86 & 0.82 & 0.80 & & & 0.850 \\
\hline \multicolumn{2}{|c|}{ Average } & 0.900 & 0.854 & 0.819 & 0.791 & 0.844 & 0.883 & \\
\hline \multirow{6}{*}{$2^{\#}$ oil sample } & 31 & 0.84 & 0.73 & 0.78 & 0.85 & 1.08 & 1.16 & 0.906 \\
\hline & 32 & 0.81 & 0.76 & 0.77 & 0.81 & 0.84 & 0.91 & 0.816 \\
\hline & 33 & 0.75 & 0.69 & 0.78 & 0.84 & 0.93 & 1.00 & 0.833 \\
\hline & 34 & 0.76 & 0.70 & 0.78 & 0.82 & 0.87 & 0.92 & 0.809 \\
\hline & 35 & 0.75 & 0.79 & 0.83 & 0.81 & 0.88 & 0.97 & 0.839 \\
\hline & 36 & 0.81 & 0.82 & 0.88 & 0.84 & 0.91 & 0.95 & 0.868 \\
\hline \multirow[t]{3}{*}{ Averc } & & 0.79 & 0.75 & 0.80 & 0.83 & 0.92 & 0.98 & \\
\hline & 28 & 0.76 & 0.72 & 0.74 & 0.65 & & & 0.717 \\
\hline & 29 & 0.77 & 0.68 & 0.72 & 0.74 & 0.79 & 1.01 & 0.784 \\
\hline \multirow[t]{3}{*}{$3^{\#}$ oil sample } & 30 & 0.76 & 0.74 & 0.75 & 0.77 & 0.79 & 0.85 & 0.777 \\
\hline & 31 & 0.81 & 0.76 & 0.76 & 0.71 & 0.81 & 0.83 & 0.780 \\
\hline & 32 & 0.67 & 0.78 & 0.78 & 0.78 & & & 0.753 \\
\hline \multicolumn{2}{|c|}{ Average } & 0.75 & 0.74 & 0.75 & 0.73 & 0.80 & 0.90 & \\
\hline
\end{tabular}

The 84 groups of experimental data for 3 kinds of crude oil, 6 rates of shear rate and 9 temperatures were fitted, and the fitting results as shown in Table 5, correlation coefficient of each group regression data whole greater than 0.8338 , and the average correlation coefficient is 0.9528 .

Figure 4 is the curve shows the hysteresis loop area changes with the temperature of the central plains crude oil. Each area of hysteresis loop were decreased with the increase of temperature but different in rate of decline, which is the lower the temperature, the greater the rate of decline. And decrease the maximum rate of the first hysteresis loop, exponentially decreasing trend and the remaining are approximately linear decline. Study results were consistent with Qifu Li [10] and other researchers. Investigate its reason, waxy crude oil form a relatively strong wax crystal structure at a low temperature, which means has a strong thixotropy, so hysteresis loop area is large. Conversely, at a high temperature, wax crystal structure was formed relatively weak, so hysteresis loop area is small.

\section{Conclusions}

1) For gelled waxy crudes oil, the thixotropic behavior of hysteresis loop which is formed under cyclic loading of linear increasing and decreasing shear rate is studied. Hysteresis loop area could represent the strength of thixotropic properties. With increasing of the rate of share rate sweep, the area of hysteresis loop increased. The area of second hysteresis loop is much smaller than first one, and began a slow decline from the third hysteresis loop.

2) Areas of each hysteresis loops were decreased with increasing temperature, but it was different in decline rate- the lower the temperature, the greater the rate of decline. The maximum rate of decrease is the first hysteresis loop with exponential decline, and remaining hysteresis hoops approximately decrease linearly.

3) We developed a relationship between the rate of shear rate and the hysteresis loop area. According to the experimental results, an empirical correlation is developed to describe the relationship of hysteresis loop area and rate of share rate sweep. 


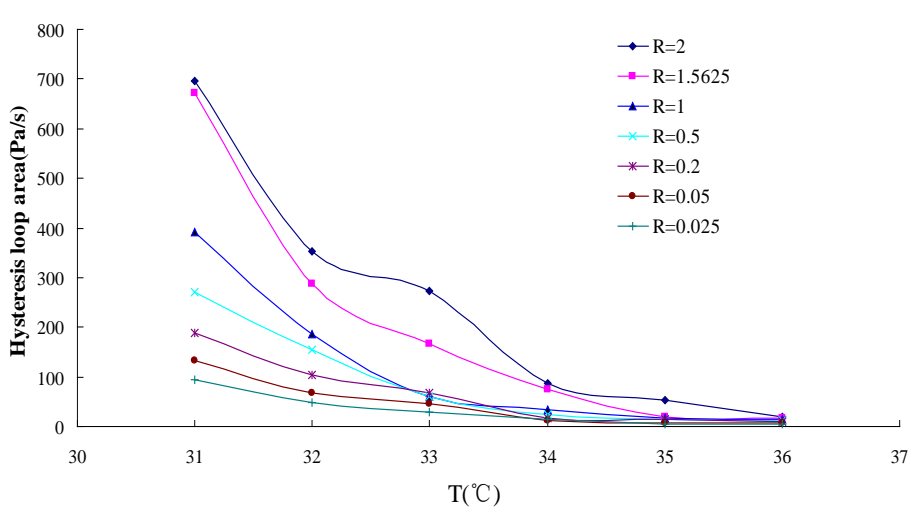

(a)

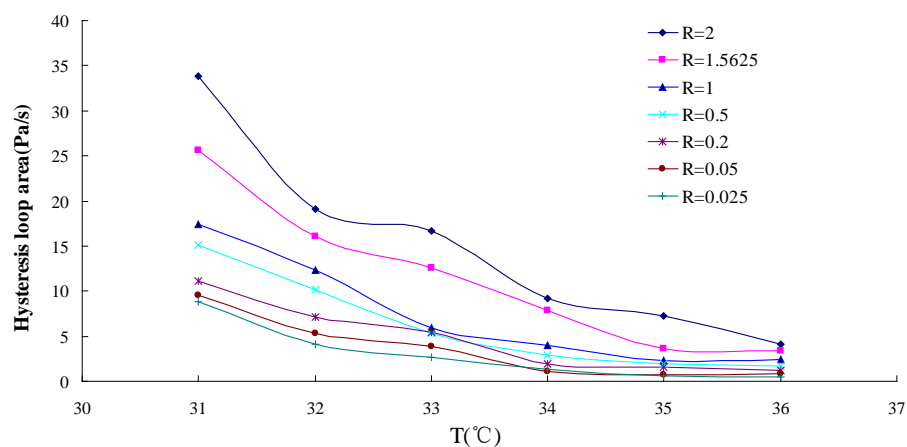

(b)

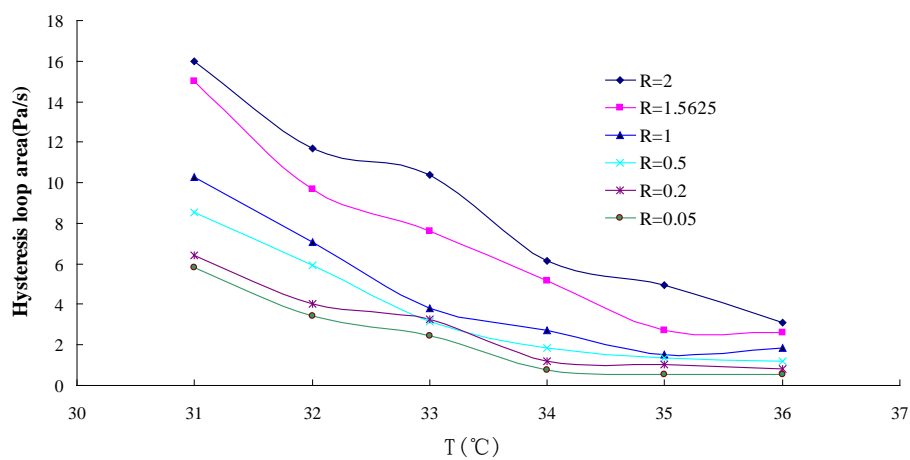

(c)

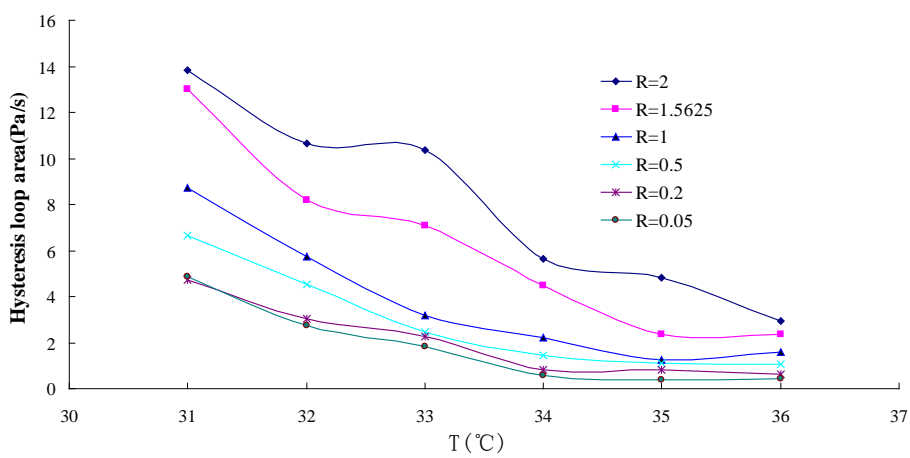

(d)

Figure 4. Experimental curve for hysteresis loop area change with temperature of central plains crude ((a) for the second hysteresis loop; (b) for the second hysteresis loop; (c) for the second hysteresis loop; (d) for the second hysteresis loop). 
Table 5. The fitting results for four kinds of oil sample.

\begin{tabular}{|c|c|c|c|c|c|}
\hline Oil sample & Temperature & $a$ & $b$ & c & Correlation coefficient \\
\hline \multirow{4}{*}{$1^{\#}$ oil sample } & 33 & 3480.43 & -3.70 & 0.365 & 0.9818 \\
\hline & 34 & 148.60 & -3.10 & 0.267 & 0.9867 \\
\hline & 35 & 79.06 & -2.72 & 0.30 & 0.9817 \\
\hline & 36 & 41.70 & -2.37 & 0.25 & 0.9856 \\
\hline \multirow{6}{*}{ 2\# oil sample } & 31 & 466.21 & -4.46 & 0.601 & 0.9710 \\
\hline & 32 & 227.32 & -3.86 & 0.53 & 0.9839 \\
\hline & 33 & 57.41 & -43.86 & 1.53 & 0.8462 \\
\hline & 34 & 45.98 & -2.88 & 0.89 & 0.9567 \\
\hline & 35 & 22.93 & -2.53 & 0.83 & 0.8338 \\
\hline & 36 & 14.88 & -2.04 & 0.35 & 0.9796 \\
\hline \multirow{5}{*}{ 3\# oil sample } & 28 & 1122.55 & -4.17 & 0.44 & 0.9874 \\
\hline & 29 & 438.15 & -4.08 & 0.28 & 0.9915 \\
\hline & 30 & 164.99 & -3.61 & 0.18 & 0.9650 \\
\hline & 31 & 68.54 & -2.99 & 0.13 & 0.9492 \\
\hline & 32 & 35.39 & -2.67 & 0.25 & 0.9922 \\
\hline
\end{tabular}

\section{Funding}

National Natural Science Foundation of China (No 51404072).

\section{References}

[1] Stokes, J.R. and Telford, J.H. (2004) Measuring the Yield Behaviour of Structured Fluids. Journal of Non-Newtonian Fluid Mechanics, 124, 137-146. http://dx.doi.org/10.1016/j.jnnfm.2004.09.001

[2] Barnes, H.A. (1997) Thixotropy Review. Journal of Non-Newtonian Fluid Mechanics, 70, 1-33. http://dx.doi.org/10.1016/S0377-0257(97)00004-9

[3] Hou, L. and Zhang, J.J. (2005) Research and Analysis of the Yield Stress of Waxy Crude Oil. Gas Storage and Transportation, 24, 5-9.

[4] Dullaert, K. and Mewis, J. (2006) A Structural Kinetics Model for Thixotropy. Journal of Non-Newtonian Fluid Mechanics, 139, 21-30. http://dx.doi.org/10.1016/j.jnnfm.2006.06.002

[5] Cheng, D.C. (1986) Yield Stress: A Time-Dependent Property and How to Measure It. Rheologica Acta, 25, $542-554$. http://dx.doi.org/10.1007/BF01774406

[6] Ruben, F.G.V., Romano, L., Emanuele, V., et al. (2005) Rheological Behaviour and Structural Interpretation of Waxy Crude Oil Gels. Langmuir, 21, 6240-6249. http://dx.doi.org/10.1021/la050705k

[7] Cheng, D.C.-H. (1987) Thixotropy. International Journal of Cosmetic Science, 9, 151-191. http://dx.doi.org/10.1111/j.1467-2494.1987.tb00472.x

[8] Mewis, J. (1979) Thixotropy-General Review. Journal of Non-Newtonian Fluid Mechanics, 6, 1-20. http://dx.doi.org/10.1016/0377-0257(79)87001-9

[9] Mewis, J. and Wagner, N.J. (2009) Thixotropy. Advances in Colloid and Interface Science, 147-148, $214-227$. http://dx.doi.org/10.1016/j.cis.2008.09.005

[10] Li, Q.F., Miao, Q., Gao, X.L., Xue, X.D., Wang, L., Yang, Z.D. and Wang, Z.L. (2011) Characterize the Thixotropic Process of Waxy Crude Oil with Hysteresis Curve Method. Oil \& Storage and Transportation, 10, 761-763+716. 